\title{
The sequence of the extruded non-template strand determines the architecture of R-loops.
}

Yeraldinne Carrasco-Salas ${ }^{1}$, Amélie Malapert ${ }^{2}$, Shaheen Sulthana ${ }^{3}$, Bastien Molcrette ${ }^{1}$, Léa ChazotFranguiadakis $^{1}$, Pascal Bernard ${ }^{2}$, Frédéric Chédin ${ }^{3}$, Cendrine Faivre-Moskalenko ${ }^{{ }^{*}}$ and Vincent Vanoosthuyse ${ }^{2^{*}}$

1 Université de Lyon, ENSL, UCBL, CNRS, Laboratoire de Physique, 46 Allée d'Italie, 69007, Lyon, France

2 Université de Lyon, ENSL, UCBL, CNRS, Laboratory of Biology and Modelling of the Cell (LBMC), 46 Allée d'Italie, 69007, Lyon, France

${ }^{3}$ Department of Molecular and Cellular Biology, University of California, Davis, California, USA, 95616

* To whom correspondence should be addressed: Vincent Vanoosthuyse (vincent.vanoosthuyse@ens-lyon.fr). Correspondence may also be addressed to Cendrine FaivreMoskalenko (cendrine.moskalenko@ens-lyon.fr). 


\begin{abstract}
Three-stranded R-loop structures have been associated with genomic instability phenotypes. What underlies their wide-ranging effects on genome stability remains poorly understood. Here we combined biochemical and atomic force microscopy approaches with single molecule Rloop footprinting to demonstrate that R-loops formed at the model Airn locus in vitro adopt a defined set of three-dimensional conformations characterized by distinct shapes and volumes, which we call R-loop objects. Interestingly, we show that these R-loop objects impose specific physical constraints on the DNA, as revealed by the presence of stereotypical angles in the surrounding DNA. Biochemical probing and mutagenesis experiments revealed that the formation of R-loop objects at Airn is dictated by the sequence of the extruded non-template strand, suggesting that $R$-loops possess intrinsic sequence-driven properties. Consistent with this, we show that R-loops formed at the fission yeast gene sum 3 do not form detectable Rloop objects. Our results reveal that R-loops differ by their architectures and that the organization of the non-template strand is a fundamental characteristic of R-loops, which could explain that only a subset of $R$-loops is associated with replication-dependent DNA breaks.
\end{abstract}

\title{
INTRODUCTION
}

DNA:RNA hybrids have emerged as important regulators of genome stability and gene expression (reviewed in (1)). One possible source of DNA:RNA hybrids in the genome is the formation of cotranscriptional R-loops, where the nascent RNA hybridizes with its DNA template through WatsonCrick interactions, whilst the non-template DNA strand remains single-stranded (reviewed in (1)). A number of DNA/RNA helicases as well as the two ribonucleases $\mathrm{H}$ (RNase $\mathrm{H} 1$ \& $\mathrm{H} 2$ ) target DNA:RNA hybrids and thereby maintain R-loop formation to low levels (reviewed in (1)). However, when these activities fail, wide-ranging evidence suggests that the resulting stabilization of R-loops interferes with the stability of replication forks during DNA replication and is associated with DNA damage and genome instability (2-5). The molecular mechanisms leading to DNA damage are still poorly understood but a recent study proposed that only a small subset of R-loop- forming regions $(\sim 10 \%)$ actually induces DNA breaks after DNA replication (2). To explain this observation, it was proposed that the chromosomal context in which R-loops form must contribute to the accumulation of DNA damage. An alternative and equally possible explanation could be that there are in fact different types of R-loops with different intrinsic properties, with some imposing greater physical constraints on the surrounding DNA than others. Here we test this possibility using Atomic Force Microscopy (AFM) to monitor the impact of R-loop formation on the surrounding DNA.

Both in vitro and in vivo observations show that the propensity of a transcription unit to form Rloops is determined at least in part by the sequence of the non-template strand. In particular, Gskewed non-template strands containing stretches of consecutive $G$ residues significantly increase the probability of forming co-transcriptional R-loops (6-8). Although strong determinants of R-loop formation have been identified, little is known about the actual architecture of R-loops. In particular, how the extruded single-stranded DNA (ssDNA) is organized and stabilized has not yet been thoroughly addressed experimentally. It is conceivable that it could recruit specific proteins and/or form secondary structures, opening up the possibility that R-loop architecture is variable and dependent on several parameters such as size and/or sequence. For instance, it was reported previously that R-loop formation at the murine $S \gamma 3$ immunoglobulin switch regions allowed the extruded non-template strand to assemble G-quadruplex structures $(9,10)$, although this is controversial as others failed to detect Gquadruplex formation in the same conditions $(11,12)$. To date, an in-depth assessment of R-loop architecture and its potential diversity is missing.

Here we use a combination of single molecule approaches to study the architecture of transcription-dependent R-loops formed in vitro. We demonstrate that the extruded non-template single-stranded DNA (ssDNA) strand determines the architecture of R-loops and their physical properties and that R-loop architecture can be manipulated using site-directed mutagenesis. This opens the possibility that the extruded ssDNA is a distinguishing feature of R-loops in vivo.

MATERIAL AND METHODS 
Plasmid preparation. The plasmids used in this study are shown on Figure S1. Airn and the Airn Gstretch mutant were synthesized (GeneCust) and cloned into pUC57. sum3 was amplified from genomic DNA using the forward primer Xba_sum3 FW that contains the sequence of the T3 promoter (5'- GCGTCTAGAATTAACCCTCACTAAAGGGAATGAGCGACAATGTACAGC- 3') and the reverse primer Bam_sum3 RV (5'- GCGGGATCCTTACCACCAGGATTGAGCAC- 3') and cloned into the pUC57 plasmid using standard protocols. pDR18 (11) containing 4 repetitions of the immunoglobulin S $\gamma 3$ switch regions was a kind gift from Dr Michael R. Lieber (University of Southern California). The

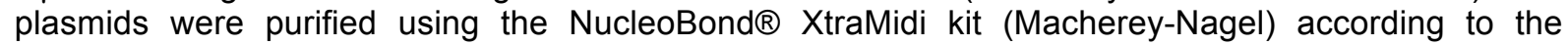
manufacturer's recommendations.

In vitro transcription of R-loops. $1875 \mathrm{ng}$ of circular plasmids were incubated at $37^{\circ} \mathrm{C}$ with $50 \mathrm{U}$ of T3 RNA Polymerase (Promega) in a transcription buffer $(40 \mathrm{mM}$ Tris- $\mathrm{HCl} \mathrm{pH} 7.9,6 \mathrm{mM} \mathrm{MgCl}, 2 \mathrm{mM}$ spermidine, $10 \mathrm{mM} \mathrm{NaCl}, 20 \mathrm{mM}$ DTT, 0,05\% Tween-20) containing 0,25 mM of rATP, rCTP, rUTP and rGTP (Promega). After 30 minutes at $37^{\circ} \mathrm{C}$, T3 was inactivated by incubating the reaction at $65^{\circ} \mathrm{C}$ for 10 minutes. When specified, the $\mathrm{NaCl}$ in the reaction buffer was replaced by $40 \mathrm{mM} \mathrm{KCl}$ or $40 \mathrm{mM}$ $\mathrm{LiCl} .750 \mathrm{ng}$ of transcribed plasmids were then incubated in the CutSmart Buffer (New England Biolabs) containing appropriate restriction enzymes and $2 \mathrm{U}$ of RNase H. pFC53 was digested with ApaLI, pUC57-Airn was digested with Kpnl and HindIII and pUC57-sum3 was digested with BamHI and Xbal. After 140 minutes at $37^{\circ} \mathrm{C}$, the $\mathrm{NaCl}$ concentration was brought to $500 \mathrm{mM}$ and $0,4 \mu \mathrm{g}$ of RNase $A$ was added for 20 minutes at $37^{\circ} \mathrm{C}$ to digest soluble RNAs. The DNA was then purified using chloroform and isopropanol precipitation and resuspended in $10 \mathrm{mM}$ Tris. The DNA was run on $0,8 \%$ agarose gels prepared in TBE $1 \mathrm{X}$ without intercalating agent.

Nuclease P1 treatment. After in vitro transcription and restriction digest, the $\mathrm{NaCl}$ concentration was brought to $500 \mathrm{mM}$ and $10 \mathrm{U}$ of nuclease P1 (reference N8630, SIGMA) was added. The mixture was incubated at $50^{\circ} \mathrm{C}$ for 45 minutes. The DNA was subsequently purified using choloroform extraction and isopropanol precipitation.

Dot blot analysis. Serial dilutions of DNA (80 ng, $40 \mathrm{ng}, 20 \mathrm{ng}, 10 \mathrm{ng}, 5 \mathrm{ng}$ ) were spotted on Hybond $^{T M}-\mathrm{N}+$ membrane (Amersham RPN203B) and cross-linked twice with UV $(0,12 \mathrm{~J})$. To quantify DNA:RNA hybrid formation, the membrane was first blocked in MES/BSA buffer (100 mM MES pH 6,6, $\mathrm{NaCl} 500 \mathrm{mM}, 0,05 \%$ Triton, $2 \mathrm{mg} / \mathrm{mL}$ BSA) for 30' at RT and then incubated overnight in 0,02 $\mu \mathrm{g} / \mathrm{mL}$ of S9.6 antibody. After extensive washes in MES/BSA buffer, exposure to a secondary antibody and ECL revelation was performed using SuperSignal West Femto Maximum Sensitivity Substrate (ThermoScientific 34096) according to standard protocols. To quantify dsDNA, the membrane was first blocked in PBS $1 \times 0,02 \%$ Tween-20 containing 5\% Milk and incubated overnight with the dsDNAspecific antibody (ab27156 Abcam, dilution 1/1000). To quantify G4 formation, the membrane was first blocked in BG4 buffer (13) (25 mM Hepes, $10 \mathrm{mM} \mathrm{NaCl}, 110 \mathrm{mM} \mathrm{KCl}, 130 \mathrm{mM} \mathrm{CaCl}, 1 \mathrm{mM} \mathrm{MgCl}$ ) containing 1\% BSA and incubated overnight with the G4-specific antibody (BG4, MABE917, Merck) at $500 \mathrm{ng} / \mathrm{mL}$ in BG4 buffer containing 1\% BSA. After extensive washings in PBS 1X 0,02\% Tween-20, the membrane was incubated for 1 hour with an anti-Flag antibody (M2, Sigma, dilution 1/1000) at room temperature, followed by exposure to a secondary antibody and ECL revelation using SuperSignal West Femto Maximum Sensitivity Substrate (ThermoScientific 34096). As a positive control, a ssDNA oligonucleotide corresponding to a G4-forming sequence of the cmyc promoter (5'GAGGGTGGGGAGGGTGGGGAAGG- 3') was incubated at $94^{\circ} \mathrm{C}$ for 5' in either $10 \mathrm{mM}$ Tris $\mathrm{pH} 7.4$, $100 \mathrm{mM} \mathrm{KCl}$ (KCl G4 buffer) or in $10 \mathrm{mM}$ Tris pH $7.4100 \mathrm{mM} \mathrm{LiCl} \mathrm{(LiCl} \mathrm{G4} \mathrm{buffer)} \mathrm{and} \mathrm{then} \mathrm{let} \mathrm{to} \mathrm{cool}$ down at room temperature for 1 hour. 30 ng of DNA (oligonucleotide or plasmid) was then spotted on the membrane.

Atomic Force Microscopy. $5 \mathrm{ng}$ of DNA were diluted in TM buffer (10 mM Tris pH 7.4, $5 \mathrm{mM} \mathrm{MgCl}_{2}$ ) and loaded on mica disks previously cleaved using Scotch tape to obtain a flat surface. After 2 minutes, the sample was washed with $1 \mathrm{~mL}$ of ultrapure water and dried gently using a nitrogen flow. The samples were imaged using an Atomic Force Microscope (Bruker, Nanoscope $V$ Multimode 8 model) using the Tapping Mode in air. Typical AFM of $3 \mu \mathrm{m}^{\star} 3 \mu \mathrm{m}\left(512 * 512\right.$ pixels $\left.{ }^{2}\right)$ were acquired at a rate of $2 \mathrm{~Hz}$ using TESP or DLCS cantilever with a resonant frequency around $300 \mathrm{kHz}$. All images were edited using NanoScope Analysis program to remove the tilt and low noise frequency in the image, before further analysis (see Supporting material).

SMRF-seq based R-loop footprinting. After in vitro transcription, the DNA was treated with sodium bisulfite under non-denaturing conditions (no denaturation and incubation at $37^{\circ} \mathrm{C}$ for $3 \mathrm{hrs}$ ) using the 
Zymo Lightning bisulfite modification kit (Zymo Research). Following sample clean-up, the DNA was amplified using native primers flanking the $\mathrm{R}$-loop prone region and Pacific Biosciences sequencing libraries were built directly from PCR amplicons. Upon library validation, sequencing was performed on pooled barcoded libraries on a Pacific Biosciences RSII instrument. Following sequencing, the Gargamel computational pipeline was used to identify SMRF footprints by tracking significant patches of $\mathrm{C}$ to $\mathrm{T}$ conversion across high quality circular consensus single reads as described in (14).

\section{RESULTS}

\section{R-loop formation at Airn allows the folding of the non-template strand into secondary structures.}

The non-template strand of the mouse Airn non-coding RNA (ncRNA) was previously shown to display a strong G-skew over roughly $1,4 \mathrm{~kb}$ and to contain several stretches of consecutive $\mathrm{G}$ residues (6). Consistent with previous observations $(4,6)$, we found that this $1,4 \mathrm{~kb}$ portion of Airn forms co-transcriptional R-loops after in vitro transcription using the bacteriophage T3 RNA Polymerase. As reported previously (6), R-loop formation on circular templates was associated with a pronounced shift in plasmid mobility on agarose gels towards the relaxed form (Figure 1A). The formation of stable and RNase H-sensitive DNA:RNA hybrids at Airn was confirmed by dot blots with the S9.6 antibody (15) (Figure 1B). When restriction enzymes were used to linearize the circular templates after in vitro transcription, R-loop formation resulted in the slower mobility of the linear template on agarose gels (Figure 1C). Strikingly, treatment with nuclease $P 1$, which specifically digests ssDNA, completely reverted this upward shift without interfering with the stability of DNA:RNA hybrids (Figure 1C\&D). These observations strongly suggest that the slower mobility induced by Rloop formation on linear Airn templates is caused by the extruded ssDNA and not by the presence of DNA:RNA hybrids. This is consistent with the idea that the formation of DNA:RNA hybrids at Airn allows the non-template strand to form secondary structures, which are responsible for the mobility shift of the linear template on agarose gels.

\section{Characterization of R-loop architecture at Airn using Atomic Force Microscopy.}

To get a better insight into the architecture of co-transcriptional R-loops formed at Airn, we used Atomic Force Microscopy (AFM) on mica surfaces to characterize the consequences of R-loop formation on the Airn template. AFM is particularly well-suited to determine with high statistics the shape and the height of individual DNA molecules at the nanometre scale $(16,17)$. Maximum R-loop formation was achieved by transcribing circular Airn templates, which were then cut using restriction enzymes to dissociate the Airn coding region (short fragment) from the plasmid backbone (long fragment). Both fragments were subsequently purified and imaged together on mica surfaces (Figure $2 \mathrm{~A})$. Strikingly, in vitro transcription resulted in the formation of regions of greater height and complex architecture on $>80 \%$ of short fragments (Figure 2A\&C). Similar objects were observed when Airn was transcribed from another plasmid, pFC53 (6) (Figure 2B). Regions of greater height were however rarely observed on the plasmid backbone, or on the Airn fragment when the DNA had not been transcribed (see below). Furthermore, their frequency was significantly reduced when transcription was followed by a treatment with RNase $H$ to disassemble DNA:RNA hybrids (Figure 2C). Taken together, these observations demonstrate that the regions of greater height observed at Airn upon transcription resulted from the formation of R-loops. Thereafter, such structures are referred to as ' $R$ loop objects'.

Using a custom-built image analysis software (see methods and Figure S2), we accurately measured the skeleton length of all the DNA molecules imaged (Figure 2D). These measurements confirmed that transcription did not affect the length distribution of the plasmid backbone, consistent with our observation that R-loop objects did not form on the plasmid backbone. On the contrary, transcription resulted in the significant shortening of the Airn fragment. Strikingly, this shortening was largely reverted after $\mathrm{RNase} H$ treatment, establishing that $\mathrm{R}$-loop objects assemble in a reversible and DNA:RNA hybrid-dependent manner. By making correlations between the volume of every object and the skeleton length of the corresponding molecule, we showed that R-loop objects of greater volume trigger greater reductions in skeleton length (Figure 2E), establishing that bigger objects contain more DNA. On the other hand, the few objects that remained after RNase $\mathrm{H}$ treatment had a significantly smaller volume (Figure $2 \mathrm{E}$ ), consistent with the observation that they only resulted in a small reduction in skeleton length (Figure 2D). Taken together, these data show that in vitro transcription of Airn resulted in the formation of R-loop objects of varying sizes and containing varying amount of DNA. 
We imaged several thousands of molecules over multiple experiments and reproducibly identified three different types of R-loop objects. We named those objects 'blobs', 'spurs' and 'loops' in keeping with the names used to describe the objects that formed after in vitro transcription of the Rloop-prone murine $S \gamma 3$ immunoglobulin switch regions (10). 'Blobs' represent R-loop objects aligned on the main axis of the DNA molecule, whilst 'spurs' come away from this axis; 'Loops' correspond to objects formed when a 'blob' sits at the base of a loop of DNA of regular height (Figure 3A). We found that 'spurs' were slightly more abundant than 'blobs' and 'loops' (Figure 3B) and had a greater volume (Figure 3C). Accordingly, 'spurs' were associated with a greater reduction in skeleton length than 'blobs' (Figure 3C) and therefore contained a greater amount of DNA (Figure 3D). 'Blobs' and 'loops' had similar volumes but 'loops' contained more DNA because they contain a loop of DNA of regular height in addition to a blob-like object. Overall, this reveals that co-transcriptional R-loops formed at the Airn locus consistently adopt three distinct types of three-dimensional architectures.

\section{R-loop objects impose local physical constraints on the surrounding DNA.}

We noticed that the presence of R-loop objects often introduced a marked angle in the DNA template, suggesting that R-loop objects mechanically constrain the surrounding DNA. To formalize this observation, we measured the distribution of angles formed between the incoming and the outgoing DNA strands for 'blob' and 'spurs' objects (see methods). We did not consider the 'loop' objects in this analysis, as it was often harder to determine the incoming and the outgoing DNA strands for this type of objects. We established that the distributions of angles for both 'blob' and 'spurs' were significantly different from the normal angle distribution determined on molecules without objects (Figure $3 E$ ), suggesting that R-loop objects kink the DNA molecule locally. Strikingly, 'blob' and 'spurs' objects introduce a different range of angles in the DNA template (respectively, $161 \pm 12$ degrees and $110 \pm 14$ degrees, mean \pm standard deviation). Importantly, this is irrespective of their volume (Figure 3E), which suggests that the internal organization of 'blob' and 'spurs' differs significantly. R-loop formation at Airn can therefore generate different types of structures, each with a distinct impact on the surrounding DNA.

To summarize, our data show that R-loop formation at Airn produces ssDNA-containing secondary structures that can be detected by AFM as objects of varying shape and volume. Those objects contain several hundreds of base pairs and introduce deformations in the surrounding DNA visible as kinks. As we detected several types of objects, it is likely that the extruded non-template strand of Airn can adopt several possible secondary structures.

\section{The position of R-loop objects overlaps with the position of R-loops mapped by SMRF-seq.}

Our observations suggested that individual transcription cycles could produce R-loops of different sequence and/or position. To accurately estimate the position of R-loop objects along the Airn template, we used our image analysis software to automatically measure the distance between each object and both DNA ends. Interestingly, the distance to the nearest end was similar between the different types of objects, whilst the distance to the furthest end was significantly greater for 'blobs' than for 'spurs' and 'loops' (Figure 4A). This observation is consistent with the idea that the starting position is conserved between objects, whilst their end position varies depending on their size.

Non-denaturing bisulfite probing can be used to map R-loops owing to the presence of bisulfite-reactive single-stranded DNA on the looped out non-template DNA strand (12). Bisulfitemediated deamination of susceptible cytosines to uracils provides a convenient readout for singlestranded DNA that is amenable to high-throughput single molecule DNA sequencing (14). Single Molecule R-loop Footprinting (SMRF-seq) can easily be used to map R-loops generated upon in vitro transcription, without prior S9.6 enrichment. For this, supercoiled pUC57-Airn was transcribed, linearized post transcription, and R-loops mapped by virtue of the strand-specific patterns of $\mathrm{C}$ to $\mathrm{T}$ conversion induced by non-denaturing bisulfite treatment. As expected, clear strand-specific patterns of bisulfite sensitivity were observed on the displaced strand of the plasmid (Figure 4B), while the RNA-paired template DNA strand was protected from bisulfite. Based on the analysis of 562 independent R-loop-carrying molecules, R-loops were distributed principally over two clusters, with $88 \%$ of R-loop footprints mapping to cluster 2 located $422-768$ bp downstream of the T3 promoter (Figure 4B). This main cluster matched well with a region of predicted high R-loop favourability (14). R-loops within cluster 2 were characterized by a discrete series of starts and stops defining overlapping molecular sub-clusters of varying sizes. The position of R-loop objects detected by AFM and the position of R-loops mapped using SMRF-seq showed striking overlap when we assumed that 
the nearest DNA end corresponded to the 5' of Airn (Figure 4C). If we assumed instead that the nearest DNA end to the object corresponded to the 3' of Airn, the overlap was poor. In addition, these data showed that the size of R-loops mapped by SMRF-seq and the size of R-loop objects detected by AFM were remarkably consistent (Figure 4C). Overall, the strong agreement between orthogonal data types, AFM mapping and chemical footprinting strongly supports the idea that R-loop objects detected by AFM correspond to genuine R-loops formed along the DNA.

\section{R-loop objects are resistant to the presence of lithium}

Similar R-loop objects were previously reported after in vitro transcription of the R-loop prone murine $S \gamma 3$ immunoglobulin switch regions (10). In this case, the formation of objects at $S \gamma 3$ repeats was attributed to the assembly of the extruded ssDNA into G-quadruplexes (G4s), because their formation was sensitive to the presence of lithium ions during transcription. To evaluate the possibility that the objects we detected at Airn were the result of G4 formation, we carried out in vitro transcription in the complete absence of $\mathrm{NaCl}$, which was replaced by either $40 \mathrm{mM} \mathrm{KCl}$ or $40 \mathrm{mM} \mathrm{LiCl}$ to respectively stabilize or destabilize G4, as previously described (10). The presence of $\mathrm{LiCl}$ had no effect on the transcription-induced shift of the DNA template on an agarose gel (Figure 5A), on the amount of DNA:RNA hybrids produced during transcription (Figure 5B) or on the abundance (Figure $5 \mathrm{C}$ ), properties and positions of the R-loop objects detected using AFM (Figure 5D\&E). Similar conclusions were reached when $\mathrm{LiCl}$ was added during transcription of pFC53 (Figure S3). To directly probe G4 formation at Airn, we used the BG4 antibody that recognizes different structural conformations of G4 (18). Dot blot assays with the BG4 antibody confirmed LiCl-sensitive G4 formation at $c-m y c$ and $\mathrm{G} 4$ formation in a plasmid containing $\mathrm{S} \gamma 3$ immunoglobulin switch regions. Treatment with nuclease P1 to digest the G4-containing ssDNA eliminated the BG4 signal at S 3 immunoglobulin switch regions. Taken together, these data highlight the specificity of the BG4 antibody, as reported previously (18). However, these experiments also established that G4 formation at Airn was not significant and importantly not induced by transcription or sensitive to RNase $H$ treatment (Figure 5F). We conclude that G4 formation is unlikely to explain the formation of R-loop objects detected by AFM at Airn.

\section{Directed mutagenesis impacts the formation of R-loop objects without interfering with the formation of DNA:RNA hybrids.}

If G4 formation does not explain the formation of R-loop objects at Airn, we considered the possibility that they resulted from the formation of other, lithium-resistant secondary structures in the extruded ssDNA. The non-template strand of Airn contains a stretch of $14 \mathrm{G}$ residues towards the 5' border of the main R-loop forming region (cluster II). To test the possibility that R-loop formation would allow this G-stretch to form secondary structures by interacting with other parts of the gene through either Watson-Crick or Hoogsteen bonds, we sought to mutagenize this motif without interfering with the R-loop forming ability of Airn. We found that the introduction of five $G$ to $A$ mutations in the $G$ stretch (Figure 6A) did not affect the amount of DNA:RNA hybrids produced after in vitro transcription (Figure 6B) but reduced dramatically the mobility shift of the linear Airn template on agarose gel (Figure 6C), in a similar way to the nuclease P1 treatment (Figure 1C). This compelling observation confirmed that the transcription-dependent and RNase $\mathrm{H}$-sensitive mobility shift of linear Airn templates on agarose gels is not due to the formation of DNA:RNA hybrids in itself but most likely to the formation of secondary structures within the extruded ssDNA. In addition, this observation strongly suggested that the stretch of $14 \mathrm{G}$ residues at the 5' of cluster II is somehow involved in the formation of these secondary structures.

In keeping with this conclusion, the G-stretch mutations affected significantly the formation of R-loop objects detected by AFM: although the mutant template was still able to produce 'blobs', 'spurs' and 'loops', their frequency (Figure 6D) and their volume (Figure 6E) were strongly reduced. In addition, the position of the remaining R-loop objects was slightly altered. In particular, 'blob' and 'loop' objects were more often detected closer to the nearest DNA end in the mutant (Figure 6F), whilst the position of the remaining 'spur' objects was largely unaffected. This new upstream position of objects overlapped with the position of the minor cluster of R-loop footprints that was seen by SMRF-seq (cluster I, Figure 4B). In support of this observation, we confirmed that the corresponding 5' end of Airn in isolation was indeed able to form R-loops and small R-loop objects (Figure S4).

Importantly, R-loop footprinting using SMRF-seq indicated that the G-stretch mutations did not dramatically affect the position of R-loops, although we detected slightly more R-loop footprints over the 5' R-loop cluster (cluster I) in the G-stretch mutant, with a corresponding decrease over the main 
cluster II (Figure 6G and Figure S5). These observations again demonstrate the strong agreement between AFM mapping data and chemical footprinting data. Altogether, our results establish that Airn contains two R-loop forming sites, among which one overlaps the G-stretch and is much more likely to harbour stable R-loops. Mutation of the G-stretch reduced slightly the likelihood of R-loop formation at this site and increased R-loop formation upstream at the secondary 5' site. More importantly, our data establish that to mutate just 5 residues in a 1375 nt-long template had a profound impact on the threedimensional R-loop architecture while causing only slight alterations in R-loops themselves as measured by their frequencies, positions and lengths.

\section{R-loop formation at sum 3 does not produce R-loop objects.}

Our in-depth analysis of R-loop formation at Airn suggested that the formation of sequencedependent secondary structures within the non-template strand contributed to the overall architecture and physical properties of R-loops. This suggested that R-loops could be distinguished by their architecture. To evaluate the potential diversity of R-loop architectures, we sought to identify a gene that would form comparable amount of R-loops as Airn upon in vitro transcription. Our previous mapping of R-loops at near nucleotide resolution in fission yeast (19) suggested that R-loops form at the sum 3 gene. Consistent with this, in vitro transcription of a circular plasmid containing sum3 resulted in the RNase $\mathrm{H}$-sensitive mobility shift of the plasmid on agarose gels towards the relaxed state (Figure 7A) and in the accumulation of DNA:RNA hybrids to levels comparable to those obtained after in vitro transcription of Airn (Figure 7B). However, when the templates were linearized, R-loop formation did not result in the upward shift of the sum3 template, contrary to what we observed on the Airn template (Figure 7A, lower panel). In addition, only very few and very small R-loop objects were detected at sum3 using AFM (Figure 7C). Consistent with this, transcription did not significantly affect the skeleton length of the sum3 template (Figure 7D). Taken together, these observations show that the extruded ssDNA at sum3 R-loops does not form detectable R-loop objects.

To conclude, our results demonstrate that R-loop formation does not necessarily lead to the formation of R-loop objects detectable by AFM and that R-loops can be distinguished by their threedimensional architecture, at least in vitro. In addition, we provide an easy way to identify objectforming R-loops: whilst all R-loops are able to relieve the supercoiling of a circular plasmid, we show here that only object-forming R-loops alter the mobility of linear templates on agarose gels.

\section{DISCUSSION}

Our multi-disciplinary analysis of R-loop formation at the single molecule resolution demonstrates that the nucleotide sequence of the extruded non-template strand of R-loops determines the three-dimensional architecture of R-loops in vitro. Consistent with this, different R-loops adopt different conformations and to mutate the DNA sequence without interfering with R-loop formation resulted in significant alterations to the R-loop objects detected by AFM. In addition, our data are consistent with the idea that at least some R-loop architectures impose significant short-range mechanical constraints on the surrounding DNA via the introduction of kinks in the template (Figure $3 E)$. More generally, our work suggests that R-loops possess intrinsic sequence-driven physical properties. Finally, in agreement with previous observations (10), we establish that there is significant variability in the size and shape of R-loop objects for a given gene, suggesting that individual transcription cycles produce R-loops of different sizes and architectures.

The formation of secondary structures within the extruded non-template strand of R-loops has long been hypothesized, mostly because at least some R-loops were shown to form over nucleotide sequences with structure-forming potential, as determined computationally $(7,20,21)$. But to our knowledge, this possibility had never been rigorously tested experimentally. In addition, a recent study reported that only $0.4 \%-9 \%$ of DNA sequences with structure-forming potential actually formed detectable secondary structures in vivo (22). Consequently, the enrichment of structure-forming motifs in R-loops does not necessarily equate to the formation of secondary structures. We therefore surmise that only very few R-loops drive the formation of biologically relevant secondary structures and a future challenge will be to identify those R-loops that genuinely drive the formation of secondary structures in vivo.

What are the R-loop objects that we revealed at Airn? It was previously proposed that similar structures formed at the $S \gamma 3$ immunoglobulin switch regions resulted from the formation of $G$ quadruplex (G4) (10). In addition, it was shown recently that there is interplay between R-loop and G4 formation, at least at a subset of R-loops (23). Although the G-stretch motif that we mutated in Airn is 
predicted to be able to form G4, there are several reasons why we do not think that G4 formation underlies the structures that we detected. First, it seems unlikely that G4 formation over a 22 nt motif would be able to trigger the assembly of $\sim 300$ nt-long structures. Second, G4 formation at this motif might be predicted to form predominantly one type of structure with a given shape and a given volume, rather than the diversity of structures that we have observed. Third, our data indicate that Rloop objects at Airn are resistant to $40 \mathrm{mM} \mathrm{LiCl}$, a treatment that should prevent the stabilization of G4s. Finally, no significant G4 formation was detected upon transcription of Airn using the G4-specific BG4 antibody. Several studies proposed that R-loop formation within a purine-rich sequence such as Airn could stabilize triplex H-DNA structures (H-loops) $(21,24,25)$. Although this is a hypothesis that would be particularly attractive to explain the formation of the loop objects that we detected (Figure $3 A$ ), it seems inconsistent with our observations: in the G-stretch mutant, the five $G$ to $A$ mutations we introduced did not change the purine content of the sequence and yet they interfered significantly with the formation of R-loop objects. Further work will be required to establish exactly the type of structures that assembles at Airn upon R-loop formation. Whatever the exact organization of the different Rloops objects that we detected, our observations strongly suggest that R-loops might also impinge on gene expression and genome stability through the formation of other types of secondary structures than G4.

The single-stranded DNA binding protein RPA was shown previously to bind to the extruded non-template strand of R-loops and to stimulate the activity of RNase H1 against R-loops (26). It is likely that the formation of secondary structures in vivo will be counter-balanced by the binding of RPA to the non-template strand and it is conceivable that the half-life of an R-loop will depend directly on this dynamic equilibrium. We therefore speculate that the formation of secondary structures within the non-template strand could stabilize R-loops by limiting RNase $\mathrm{H} 1$ access. The fact that structureforming R-loops could be more stable might contribute to their function and/or toxicity. When the enzymes that disassemble R-loops are defective, R-loops could have more time to form secondary structures, which could in turn alter their toxic potential and trigger genome instability. This model is consistent with the idea that only a small subset of R-loops is associated with DNA breaks (2). We speculate that structured R-loops could be more toxic for the cell and become replication impediments, as proposed recently (21). Consistent with this hypothesis, the formation of R-loops at Airn was shown to induce replication stress (4) and structure forming non-B DNA sequences are prominent barriers to replication fork progression $(21,27)$. Structured R-loops could impede directly the passage of replication forks. Alternatively, their consequences on the mechanical properties of the surrounding DNA could signal the presence of R-loops to the cell and, depending on the structures, recruit different proteins, which in turn could contribute to their toxicity. For example, it will be interesting to test whether or not structured R-loops represent better substrates for the flap endonucleases XPF and XPG that have been shown to contribute to the genotoxicity of R-loops (28). Alternatively, it was proposed that the toxicity of R-loops could be mediated at least in part by their ability to induce local alterations to the epigenome $(29,30)$. It is conceivable that structure-forming Rloops could have a different impact on the epigenome by recruiting different chromatin modifiers.

Finally, our data are consistent with the idea that highly structured R-loops could require more than one enzyme for complete disassembly: a DNA helicase to remove the secondary structures within the extruded ssDNA and a DNA:RNA helicase or RNase $\mathrm{H}$ to resolve the DNA:RNA hybrid itself (Figure S6). This could explain why we still detected small R-loop objects at Airn after RNase $\mathrm{H}$ treatment, even in the absence of detectable DNA:RNA hybrids (Figure 2). Alternatively, the complete removal of structure-forming R-loops might require specific helicases that can act against both DNA:RNA hybrid and DNA hairpins. It is unclear at this stage whether any of the helicases that have been implicated in R-loop resolution are also involved in removing secondary structures within the extruded non-template strand of R-loops. Most published evidence suggests that such helicases are more likely to target the DNA/RNA hybrid moiety of R-loops. It is however interesting to note that the FANCI/FANCD2 heterodimer was recently shown to bind to the non-template strand of G-rich R-loops in vitro and it was proposed that it might facilitate R-loop resolution by recruiting downstream effectors (31). It is still unclear at this stage however whether the binding of FANCI/FANCD2 heterodimers to Rloops would be influenced by secondary structures within the G-rich non-template strand. Future experiments should determine whether the proteome associated with R-loops differs whether or not their extruded non-template strand can form secondary structures.

\section{SUPPLEMENTARY DATA}


Supplementary Data are available at NAR online.

\section{FUNDING}

This work was supported by a "Chaire d'Excellence" awarded by the Agence Nationale pour la Recherche (ANR, Project TRACC, CHX11) to VV, by a "Projet Pluri-Equipe" (PPE 2016-2018) awarded by la Ligue contre le Cancer, comité du Rhône to VV, and by a "Projet Emergent" de l'ENSLYON (2016-2018) awarded to CM and VV. Work in the Chédin laboratory was supported by a grant from the National Institutes of Health (GM120607). Funding for open access charge: La Ligue contre le Cancer.

\section{CONFLICT OF INTEREST}

The authors wish to declare no conflict of interest.

\section{ACKNOWLEDGMENTS}

We thank Pénélope Legros for her technical help at the beginning of this project and Dr Michael R. Lieber for reagents. We are grateful to Stella Hartono for her help with the bioinformatics analysis of SMRF-seq results. We thank Julien Gros for helpful discussions.

\section{REFERENCES}

1. Santos-Pereira JM, Aguilera A (2015) R loops: new modulators of genome dynamics and function. Nat Rev Genet 16(10):583-597.

2. Costantino L, Koshland D (2018) Genome-wide Map of R-Loop-Induced Damage Reveals How a Subset of R-Loops Contributes to Genomic Instability. Mol Cell 71(4):487-497.e3.

3. García-Rubio M, et al. (2018) Yra1-bound RNA-DNA hybrids cause orientation-independent transcription-replication collisions and telomere instability. Genes Dev 32(13-14):965-977.

4. Hamperl S, Bocek MJ, Saldivar JC, Swigut T, Cimprich KA (2017) Transcription-Replication Conflict Orientation Modulates R-Loop Levels and Activates Distinct DNA Damage Responses. Cell 170(4):774-786.e19.

5. Lang KS, et al. (2017) Replication-Transcription Conflicts Generate R-Loops that Orchestrate Bacterial Stress Survival and Pathogenesis. Cell 170(4):787-799.e18.

6. Ginno PA, Lott PL, Christensen HC, Korf I, Chédin F (2012) R-loop formation is a distinctive characteristic of unmethylated human CpG island promoters. Mol Cell 45(6):814-825.

7. Kuznetsov VA, Bondarenko V, Wongsurawat T, Yenamandra SP, Jenjaroenpun P (2018) Toward predictive R-loop computational biology: genome-scale prediction of R-loops reveals their association with complex promoter structures, G-quadruplexes and transcriptionally active enhancers. Nucleic Acids Res 46(15):7566-7585.

8. Roy D, Lieber MR (2009) G clustering is important for the initiation of transcription-induced Rloops in vitro, whereas high $\mathrm{G}$ density without clustering is sufficient thereafter. Mol Cell Biol 29(11):3124-3133.

9. Duquette ML, Handa P, Vincent JA, Taylor AF, Maizels N (2004) Intracellular transcription of G-rich DNAs induces formation of G-loops, novel structures containing G4 DNA. Genes Dev 18(13):1618-1629.

10. $\quad$ Neaves KJ, Huppert JL, Henderson RM, Edwardson JM (2009) Direct visualization of Gquadruplexes in DNA using atomic force microscopy. Nucleic Acids Res 37(18):6269-6275.

11. Roy D, Yu K, Lieber MR (2008) Mechanism of R-loop formation at immunoglobulin class switch sequences. Mol Cell Biol 28(1):50-60.

12. Yu K, Chedin F, Hsieh C-L, Wilson TE, Lieber MR (2003) R-loops at immunoglobulin class switch regions in the chromosomes of stimulated B cells. Nat Immunol 4(5):442-451.

13. Hänsel-Hertsch R, Spiegel J, Marsico G, Tannahill D, Balasubramanian S (2018) Genomewide mapping of endogenous G-quadruplex DNA structures by chromatin immunoprecipitation and high-throughput sequencing. Nat Protoc 13(3):551-564.

14. Stolz R., et al. The interplay between DNA sequence and negative superhelicity drives R-loop structures. Proc Natl Acad Sci USA in press.

15. Boguslawski SJ, et al. (1986) Characterization of monoclonal antibody to DNA.RNA and its application to immunodetection of hybrids. J Immunol Methods 89(1):123-130. 
16. Montel F, et al. (2011) RSC remodeling of oligo-nucleosomes: an atomic force microscopy study. Nucleic Acids Res 39(7):2571-2579.

17. Japaridze A, et al. (2017) Spatial organization of DNA sequences directs the assembly of bacterial chromatin by a nucleoid-associated protein. J Biol Chem 292(18):7607-7618.

18. Biffi G, Tannahill D, McCafferty J, Balasubramanian S (2013) Quantitative visualization of DNA G-quadruplex structures in human cells. Nat Chem 5(3):182-186.

19. Hartono SR, et al. (2018) The Affinity of the S9.6 Antibody for Double-Stranded RNAs Impacts the Accurate Mapping of R-Loops in Fission Yeast. J Mol Biol 430(3):272-284.

20. Chen L, et al. (2017) R-ChIP Using Inactive RNase H Reveals Dynamic Coupling of R-loops with Transcriptional Pausing at Gene Promoters. Mol Cell 68(4):745-757.e5.

21. Šviković S, et al. (2018) R-loop formation during S phase is restricted by PrimPol-mediated repriming. EMBO J. doi:10.15252/embj.201899793.

22. Kouzine F, et al. (2017) Permanganate/S1 Nuclease Footprinting Reveals Non-B DNA Structures with Regulatory Potential across a Mammalian Genome. Cell Syst 4(3):344-356.e7.

23. De Magis A, et al. (2019) DNA damage and genome instability by G-quadruplex ligands are mediated by $\mathrm{R}$ loops in human cancer cells. Proc Natl Acad Sci U S A 116(3):816-825.

24. Grabczyk E, Fishman MC (1995) A long purine-pyrimidine homopolymer acts as a transcriptional diode. J Biol Chem 270(4):1791-1797.

25. Neil AJ, Liang MU, Khristich AN, Shah KA, Mirkin SM (2018) RNA-DNA hybrids promote the expansion of Friedreich's ataxia (GAA)n repeats via break-induced replication. Nucleic Acids Res 46(7):3487-3497.

26. Nguyen HD, et al. (2017) Functions of Replication Protein A as a Sensor of R Loops and a Regulator of RNaseH1. Mol Cell 65(5):832-847.e4.

27. Shastri N, et al. (2018) Genome-wide Identification of Structure-Forming Repeats as Principal Sites of Fork Collapse upon ATR Inhibition. Mol Cell. doi:10.1016/j.molcel.2018.08.047.

28. Sollier J, et al. (2014) Transcription-coupled nucleotide excision repair factors promote R-loopinduced genome instability. Mol Cell 56(6):777-785.

29. Castellano-Pozo M, et al. (2013) R loops are linked to histone H3 S10 phosphorylation and chromatin condensation. Mol Cell 52(4):583-590.

30. García-Pichardo D, et al. (2017) Histone Mutants Separate R Loop Formation from Genome Instability Induction. Mol Cell 66(5):597-609.e5.

31. Liang Z, et al. (2019) Binding of FANCI-FANCD2 Complex to RNA and R-Loops Stimulates Robust FANCD2 Monoubiquitination. Cell Rep 26(3):564-572.e5.

\section{FIGURE LEGENDS}

Figure 1: R-loop formation at Airn allows the folding of the non-template strand into secondary structures in vitro. (AB) The circular plasmid pUC57-Airn was transcribed in vitro and treated or not with RNase $\mathrm{H}$ as indicated. After purification, the DNA was run on agarose gels (A) or spotted on a membrane to perform dot blot analysis with the indicated antibodies (B). (CD) The circular plasmid pUC57-Airn was transcribed in vitro before restriction enzymes were used to separate the plasmid backbone from the Airn gene. After restriction digest, the reactions were treated either with $\mathrm{RNase} H$ or nuclease P1 as indicated. After purification, the DNA was run on an agarose gel (C) or spotted on a membrane to perform dot blot analysis with the indicated antibodies (D).

Figure 2: AFM analysis of the R-loop dependent secondary structures formed at Airn. (A) pUC57-Airn were processed as in Figure $1 \mathrm{C}$ and visualized using AFM. In pUC57-Airn, Airn is present on the short fragment. The blue square highlights a long DNA fragment corresponding to the plasmid backbone and white squares highly short DNA fragments corresponding to Airn. Magnifications of the molecules identified by a white square are shown underneath. (B) (left) pFC53 plasmids were processed as in Figure $1 \mathrm{C}$ and visualized using AFM. In pFC53, Airn is present on the long fragment. (right) Examples of long DNA fragments with R-loop objects. (C) After AFM imaging of pUC57-Airn, the probability of short molecules showing R-loop objects was established $(n=4$ independent experiments; error bars represent the standard deviation). The total number of short fragment molecules counted is indicated. (D) Probability density of the DNA skeleton length of all the molecules analysed (both long and short fragments) in the indicated conditions (with and without objects). (E) 2D probability density plots showing the correlation between the skeleton length and the object's volume in the indicated conditions. 
Figure 3: R-loop formation at Airn imposes mechanical constraints on the surrounding DNA. (A) Typical AFM images showing three different types of R-loop objects: blobs, spurs and loops. (B) Probability of the different types of R-loop objects ( $n=4$ independent experiments; error bars represent the standard deviation). (C) $2 \mathrm{D}$ probability density plots showing the correlation between the skeleton length and the object's volume for each category of R-loop objects. The respective 1D projections of each probability density function are also shown. (D) Estimated amount of DNA caught in each type of R-loop objects (mean \pm standard error). (E) 2D probability density plots showing the correlation between the angle and the object's volume for 'blobs' and 'spurs'. The angle formed between the DNA strand going into the object and the DNA strand coming out of the object was measured for 'blobs' (purple) and 'spurs' (green). The distribution of angles for molecules without objects was also established (yellow, see methods).

Figure 4: Position of R-loop objects on Airn. (A) Distance to the nearest DNA end (purple) and to the furthest DNA end (orange) measured for each R-loop object and represented as boxplots. These distances are reported schematically on the Airn template. The notches in the boxplots indicate the $5 \%$ uncertainty mark around the median. P-values were determined using the Wilcoxon Mann Whitney statistical test $\left(^{* * *}, p<0,001 ;{ }^{* *}, p<0,01\right)$. (B) Footprints obtained by SMRF-seq on the non-template DNA strand. Each horizontal line corresponds to a single independent DNA molecule carrying an Rloop footprint (562 overall). The position of cytosines along the amplicon is shown by vertical orange lines. The status of each cytosine after non-denaturing bisulfite probing is color-coded as indicated in the inset; R-loop footprints are indicated by red horizontal patches. Footprints were clustered by position and clusters are highlighted using color shading. The position of the different clusters relative to the T3 promoter sequence is schematized underneath together with the aggregate $\mathrm{C}$ to $\mathrm{T}$ conversion frequency derived from R-loop peaks on the non-template DNA strand. (C) The position of R-loop footprints was compared to the positions of R-loop objects detected by AFM. The overlap between the two sets of measurements is best when one assumes that the nearest DNA end represents the 5' of Airn.

Figure 5: R-loop objects at Airn do not result from G-quadruplex formation. (AB) The circular plasmid pUC57-Airn was transcribed in vitro in the presence or not of $40 \mathrm{mM} \mathrm{LiCl}$, before restriction enzymes were used to separate the plasmid backbone from the Airn template. After purification, the DNA was run on an agarose gel (A) or spotted on a membrane to perform dot blot analysis with the indicated antibodies (B). (C) Probability of R-loop objects. (D) 2D probability density plots showing the correlation between the skeleton length and the object's volume in the indicated conditions. The respective $1 \mathrm{D}$ projections of the probability density function are also shown. (E) Distance between Rloop objects and the nearest DNA end in the indicated conditions and for the different types of objects. (F) Dot blot analysis of in vitro transcription products of pDR18-S $\gamma 3$ or pUC57-Airn with the indicated antibodies. As expected, the G4 signals disappear upon treatment with nuclease P1 and G4 formation at $c-m y c$ is disrupted in the presence of $\mathrm{LiCl}$. See text and methods for details.

Figure 6: The formation of R-loop objects at Airn is dictated by the DNA sequence. (A) Schematic representation of the mutated Airn locus. (BC) The indicated circular plasmids were transcribed in vitro before restriction enzymes were used to separate the plasmid backbone from the Airn gene. After purification, the DNA was spotted on a membrane to perform dot blot analysis with the indicated antibodies (B) or run on an agarose gel (C). (D) Probability of R-loop objects (mean \pm standard deviation, $n=2$ ). (E) 2D probability density plots showing the correlation between the skeleton length and the object's volume in the indicated conditions. (F) R-loop footprinting in the G-stretch mutant. The graph displays the aggregate bisulfite conversion along the Airn sequence. The histograms quantify the percentages of footprints overlapping with cluster I and II in the two conditions. (G) Distance between R-loop objects and the nearest DNA end in the indicated conditions and for the different types of objects.

Figure 7: R-loop formation at sum3 does not result in the formation of R-loop objects. (A) The indicated circular plasmids were transcribed in vitro and treated or not with RNase $\mathrm{H}$ and run on agarose gel before (top) or after (bottom) restriction enzymes were used to separate the plasmid backbone from the indicated gene. (B) The reactions from (A) were spotted on a membrane to perform dot blot analysis with the indicated antibodies. (C) AFM imaging of pUC57-sum3 transcribed in the indicated conditions after restriction enzymes were used to separate the plasmid backbone from sum3. sum 3 corresponds to the short fragment. (D) Probability density of the DNA skeleton length of all the molecules analysed in the indicated conditions. 
Not transcribed (NT)
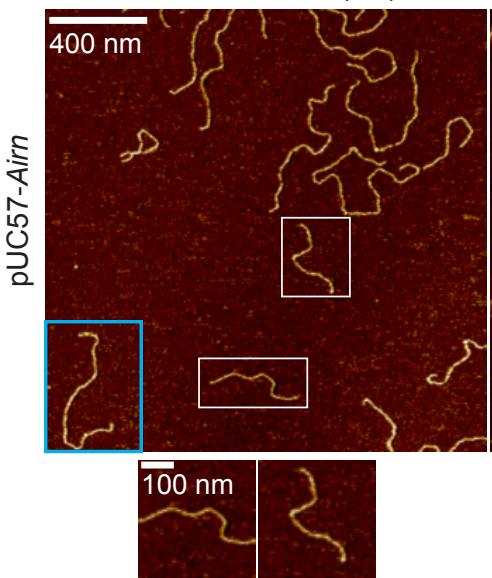

B

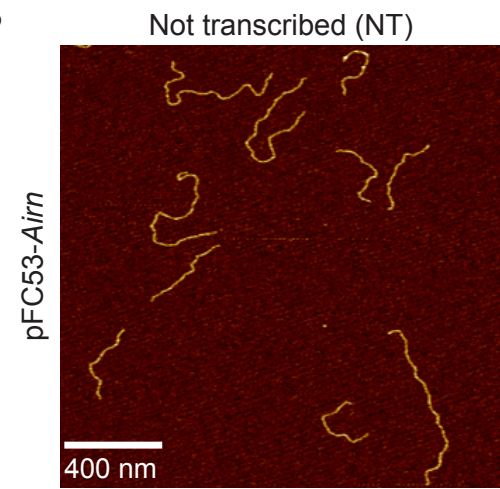

C

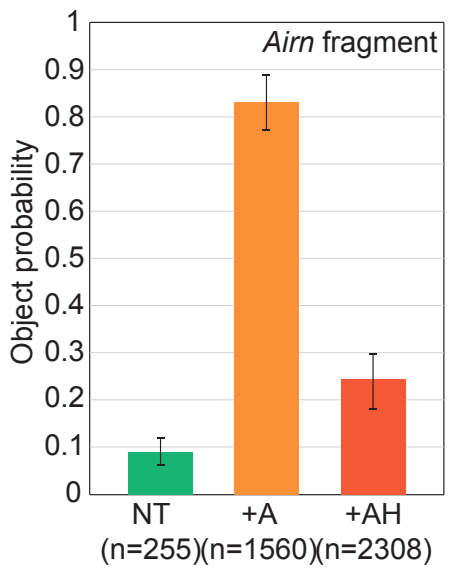

Transcribed (+A)
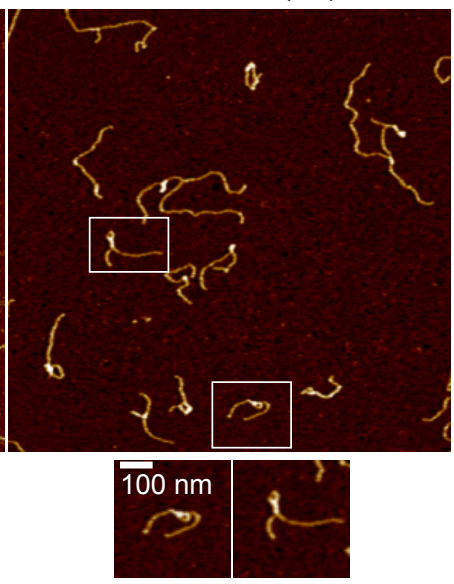

Transcribed (+A)

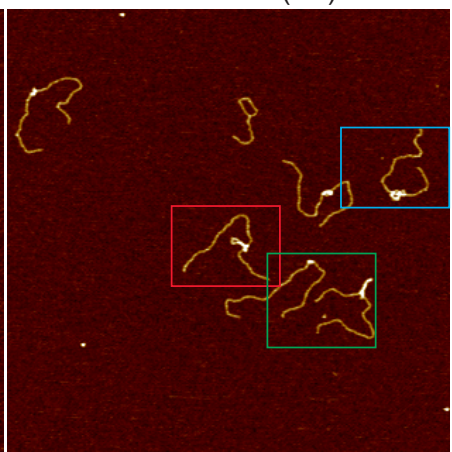

D

Transcribed+ RNase $\mathrm{H}(+\mathrm{AH})$

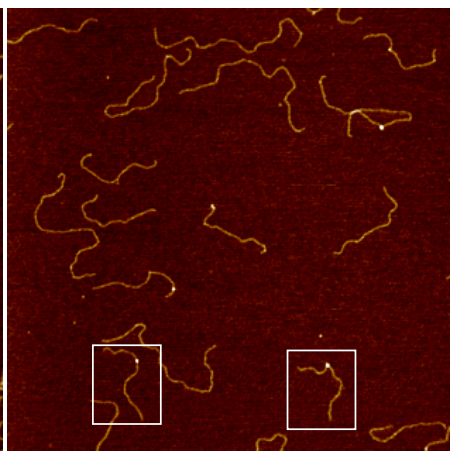

$1.0 \mathrm{~nm}$

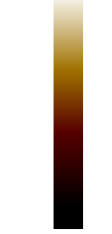

$-0.5 \mathrm{~nm}$

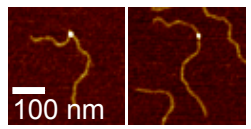

Transcribed+ RNase $\mathrm{H}(+\mathrm{AH})$

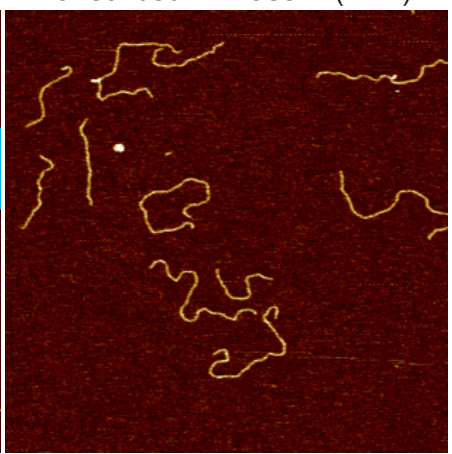

Transcribed (+A)

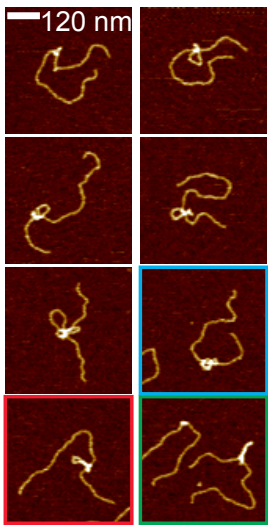

E
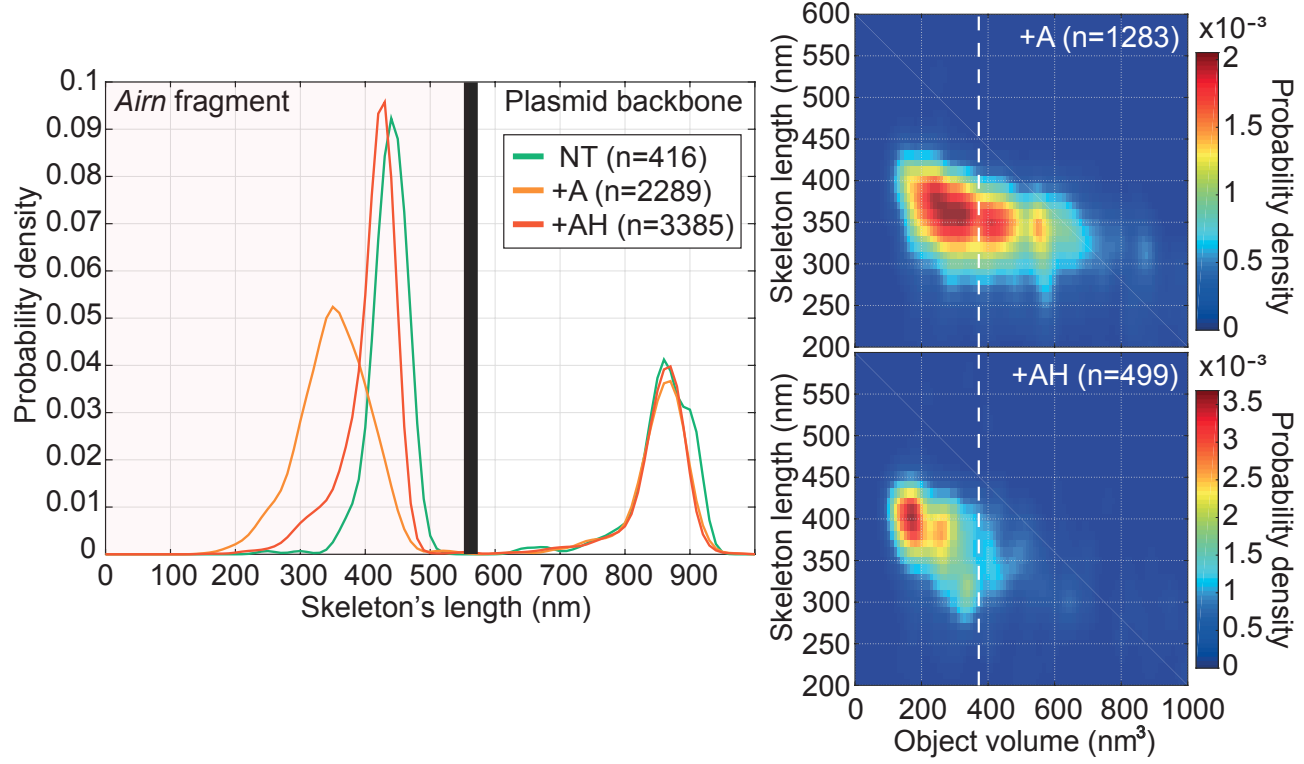
A
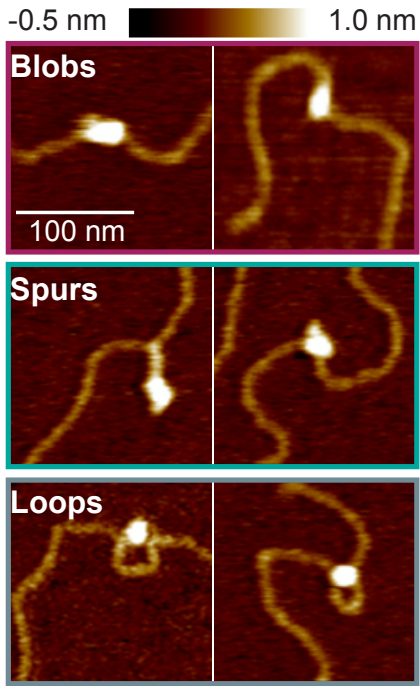

B

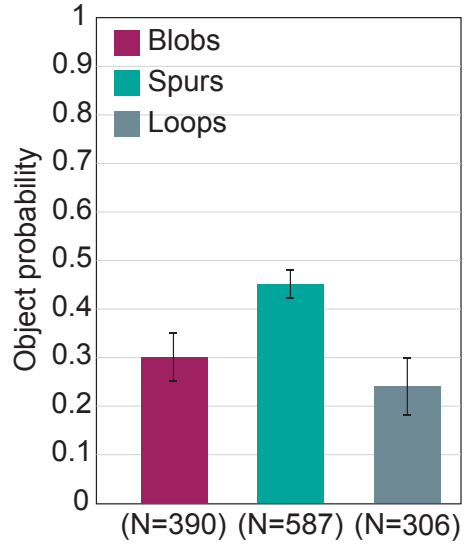

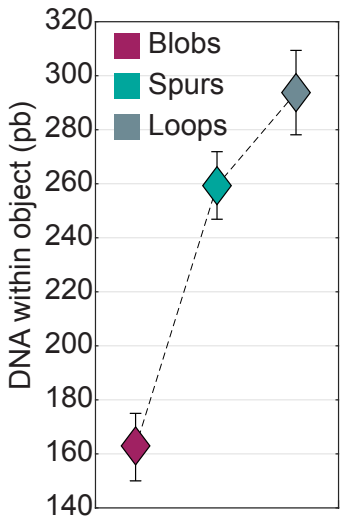

C Probability density
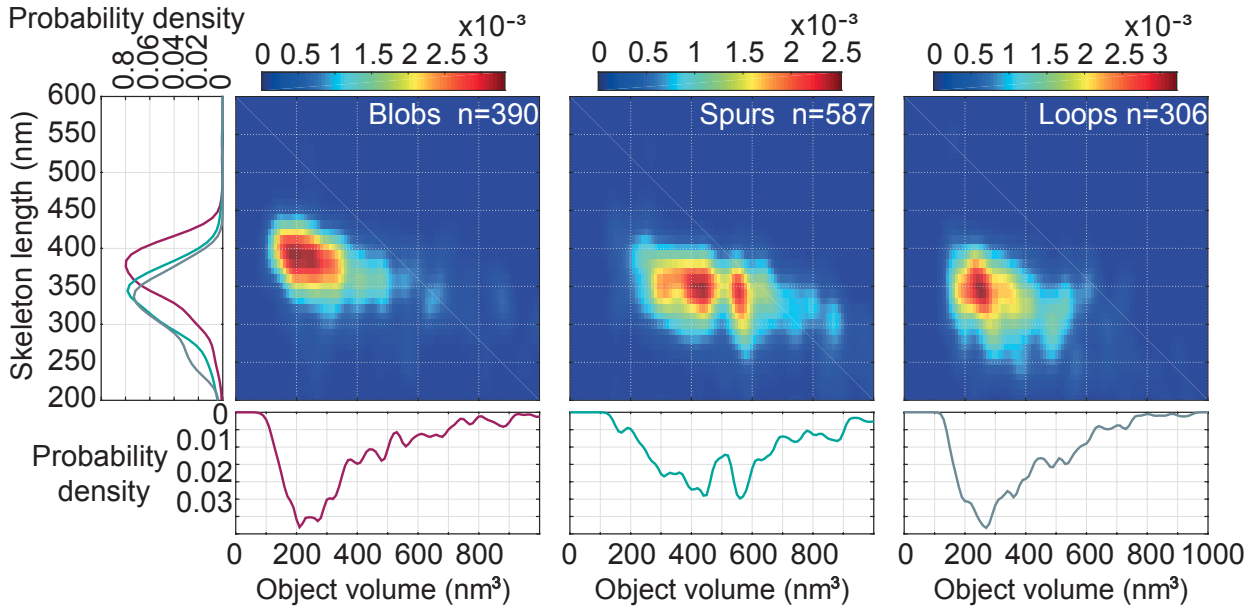

E

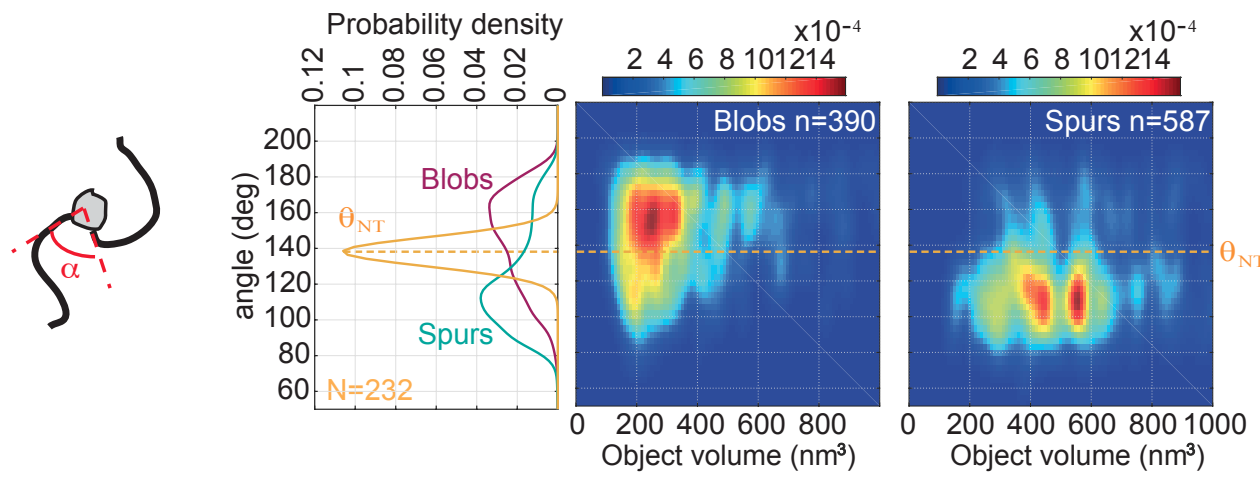




\section{B}
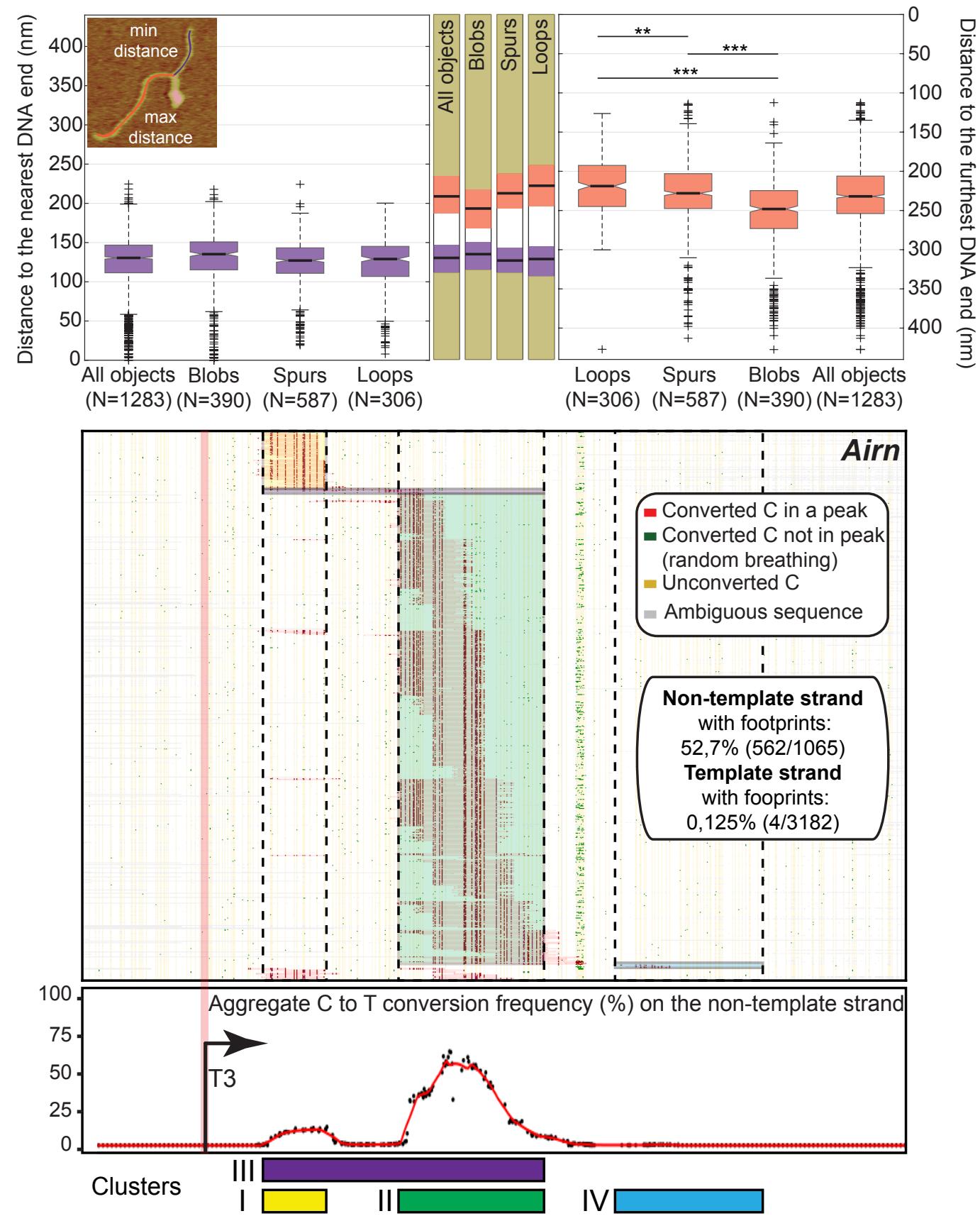

C

\ AIRN (Mouse chr 17: \ main R-loop 12741330-12742700) footprint
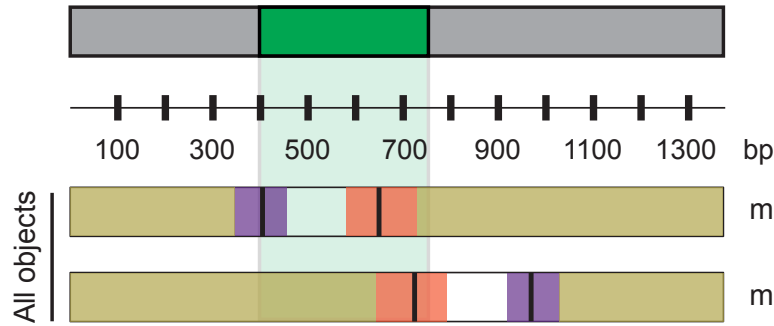

min distance $=5$, of Airn

min distance $=3$, of Airn 
